\title{
CONCEPÇÃO DE UM DISPOSITIVO PARA A REINTEGRAÇÃO DE PESSOAS COM TETRAPLEGIA INCOMPLETA AO TÊNIS
}

Abner Pereira (abner.pereira23@gmail.com) - Universidade de Brasília/ Faculdade de Tecnologia Ana Luisa Oliveira da Nóbrega costa (analuisaonc@gmail.com) - Universidade de Brasília/ Faculdade de Tecnologia

Hugo Faria Frota (hugofrota@gmail.com) - Universidade de Brasília/ Faculdade de Tecnologia

Miguel Alves Pereira Júnior (migueljr.prod@gmail.com) - Universidade de Brasília/ Faculdade de Tecnologia

Rafaela de Faria Dantas (afaela1996dantas@gmail.com) - Universidade de Brasília/ Faculdade de Tecnologia

Tiago Camargo Alves (tiago.camargoalves@gmail.com) - Universidade de Brasília/ Faculdade de Tecnologia/ Laboratório Aberto de Brasília

Andrea Cristina dos Santos (andreakieck@gmail.com) - Universidade de Brasília/ Faculdade de Tecnologia/ Laboratório Aberto de Brasília

\section{RESUMO}

Este trabalho tem como objetivo apresentar as fases iniciais do PDP (Processo de Desenvolvimento de Produtos) para a concepção de uma tecnologia assistiva que permita que pessoas com tetraplegia incompleta pratiquem o tênis paraolímpico com maior desempenho e conforto. Trata-se de um estudo de caso realizado na Associação de Centro de Treinamento de Educação Física Especial (CETEFE). A pesquisa identificou que a principal barreira enfrentada pelos jogadores de tênis de cadeira de rodas era a dificuldade de segurar, manter e transmitir força à raquete ao longo do jogo. A partir desta problemática, foram aplicados os métodos e técnicas das fases iniciais do PDP. Como resultado, obteve-se uma modelagem do produto em CAD e um protótipo impressão em 3D.

Palavras chave: desenvolvimento de produto; tecnolologia assistiva; tênis paraolímpico; tetraplegia incompleta; tetraplegia; esporte; reintragração. 


\section{INTRODUÇÃO}

O tênis quadriplégico para cadeira de rodas foi fundado em 1976 e é considerado um dos esportes em cadeira de rodas que mais cresce no mundo. De 1992 até 2016 o Tour de Tênis em Cadeira de Rodas da Federação Internacional de Tênis aumentou de 11 para 160 eventos internacionais (ALDEN, 2016). Quanto mais os esportes adaptativos crescem e evoluem, os atletas adaptados para cadeira de rodas continuam a melhorar seu desempenho (CALDWELL, 2018).

Porém, lesões de atletas de tênis nos membros superiores são comumente relatadas, na qual ocorrem $20 \%$ na mão, seguido pelo ombro $(15,5 \%)$, dedos $(11,1 \%)$ e braço $(10 \%)$. Segundo Caldwell (2018), a taxa de incidência de lesões em tênis de cadeira de rodas é de 12,7\%, na qual 37\% são lesões agudas e 47\% lesões por uso excessivo. Caldwell (2018) afirma ainda que nas paraolimpíadas de 1992, 75\% dos jogadores de tênis de cadeira de rodas adquiriram uma lesão durante o treinamento ou competição (CALDWELL, 2018).

$\mathrm{O}$ aperto da raquete, portanto, é determinante nas jogadas de tênis e a maneira como a raquete é segurada influencia o ângulo da face da raquete, a posição na qual a bola se encontra em relação ao corpo e especialmente os movimentos que ocorrem quando o impacto entre a bola e a raquete acontecem (USPTA, 2018). Muitos atletas de tênis quadriplégico para cadeira de rodas possuem dificuldade no movimento de aperto da raquete (CALDWELL, 2018), que pode ser causado por diferentes motivos, tais como poliomielite, focomelia, miopatia ou distrofia muscular ou neuropatia (ITF, 2018). Isto impede que os atletas consigam realizar o movimento de agarra e fechar completamente a mão para segurar a raquete.

Dessa forma, mesmo com a crescente relevância do esporte, os praticantes ainda utilizam de soluções rudimentares para resolver esse problema, como amarrar fitas, faixas, durex e overgrip para enfaixar as mãos. Trata-se de um improviso e apesar de baratas, causam alto desconforto e podem acarretar em inchaço, além de possibilitar o surgimento de dermatites, ocasionando desconforto e demandando longo tempo de preparo/adaptação à raquete (CARVALHO et al., 2005).

As pesquisas e o desenvolvimento tecnológico aplicado ao tênis se concentram nos materiais esportivos comerciais (roupas, bolas, raquetes, etc.) com fins lucrativos. Com isso, pouco se investe na solução de problemas de atletas paraolímpicos (CARVALHO, et al., 2005). 
A pesquisa busca responder à pergunta: como pode ser desenvolvido um projeto de produto para solucionar o problema de agarra e transmissão de força à raquete ao longo do jogo pelos praticantes com tetraplegia incompleta de tênis de cadeira de rodas?

O principal objetivo deste trabalho é, portanto, desenvolver um projeto de produto de tecnologia assistiva para jogadores de tênis tetraplégicos que jogam na modalidade Quad, classificada como tênis para cadeirantes com algum problema na mão.

Este artigo está estruturado em 5 seções. A seção 2 da pesquisa traz os conceitos teóricos concernentes ao processo de desenvolvimento do produto e tênis paraolímpico Quad. A seção 3 apresenta a metodologia da pesquisa, a seção 4 discorre sobre os resultados identificados e a seção 5 descreve as considerações finais, propostas de melhoria e sugestões para futuros trabalhos.

\section{REVISÃO TEÓRICA}

2.1 Processo de desenvolvimento de produto

O processo de desenvolvimento de produto consiste em um conjunto de atividades no qual, a partir das necessidades do mercado, restrições tecnológicas e estratégias competitivas e de produto da empresa, busca-se chegar às especificações de projeto de um produto e seu processo de produção (ROZENFELD et al., 2006).

Além de constituir uma estratégia competitiva liderada por produtos, sustentabilidade ambiental, que começa com a percepção de uma oportunidade de mercado e termina na produção, venda e entrega de um produto (ULRICH et al., 2004), o processo de desenvolvimento de produtos possui vários problemas no progresso da teoria e conceitos (SHAW, 1995).

Muitos dos problemas nessa área são derivados da falta de estudos teóricos embasados em um método sistemático (CONFORTO, 2011). Além disso, Hart evidencia a importância de investigar e compreender o corpo de conhecimento de maneira ampla antes de iniciar uma pesquisa (HART, 1998). Nesse ínterim, a definição do problema é o ponto de partida da revisão bibliográfica no processo de desenvolvimento de produtos (CONFORTO, 2011). 


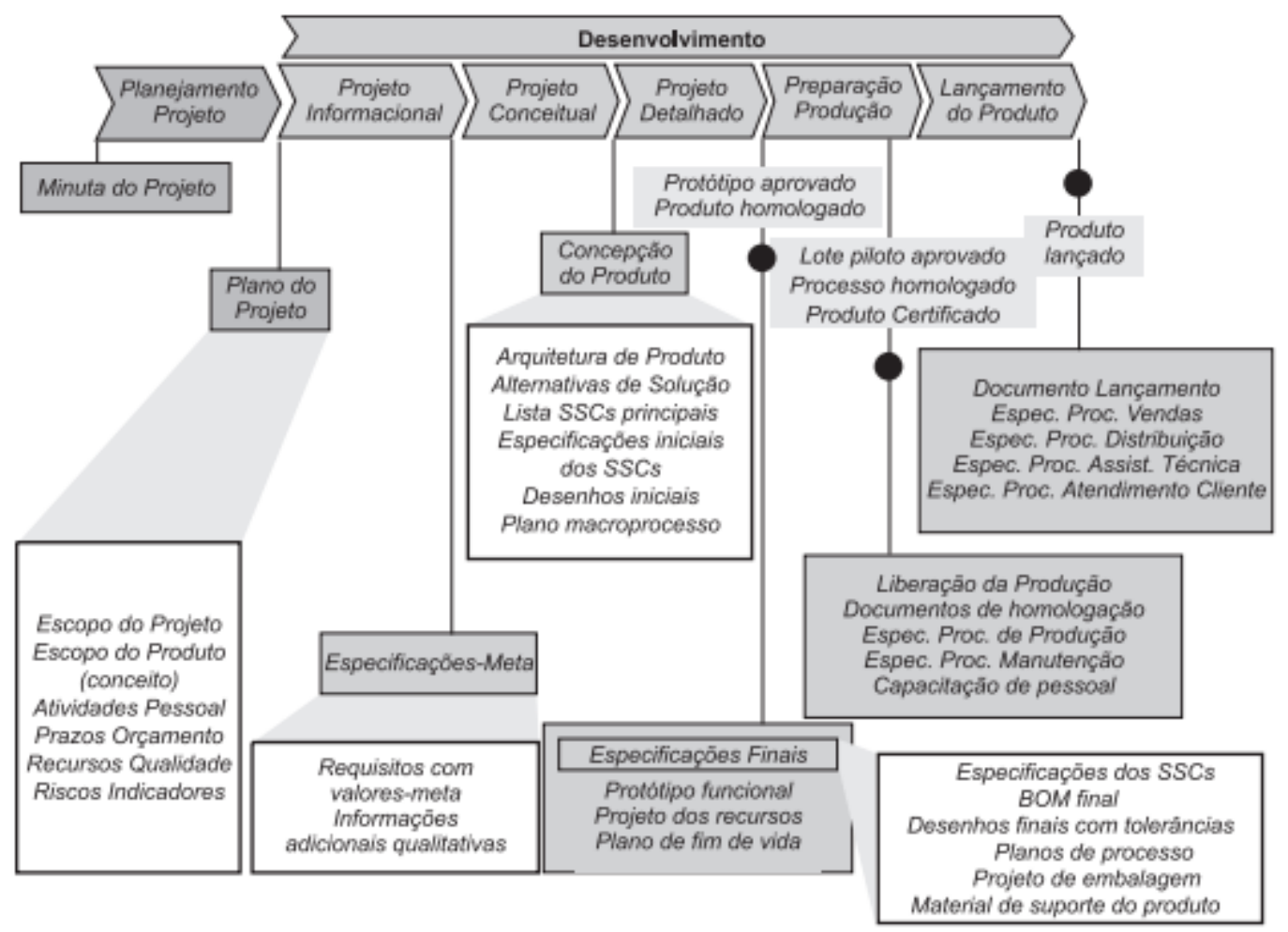

Figura 1 - Principais resultados das fases do PDP. Fonte: Rozenfeld et al. (2006, p. 48)

As principais fases do processo de desenvolvimento de produto e os resultados esperados de cada etapa são ilustrados na Figura 1.

Segundo Bardales (2013), o processo de desenvolvimento de um produto inicia-se com uma ideia ou uma demanda do cliente interno e posteriormente a equipe comercial inicia a fase de prospecção e sondagem com clientes externos e possíveis interessados. Após isso, são identificados os requisitos do cliente que se transformam em especificações de produto. Em seguida, inicia-se o processo de emissão do parecer técnico e econômico sobre o desenvolvimento do produto, com a estimativa de custos, além da definição do escopo do projeto e escopo do produto (BARDALES, 2013).

A definição de custo meta, pode ser realizada de duas maneiras: baseada no mercado ou baseada no custo. Assim, quando já existe um produto parecido no mercado, o custo meta é calculado através do levantamento de dados e quando não existe um produto similar, este, é calculado através da estimativa de custo (BARDALES, 2013). 
O projeto informacional tem como objetivo desenvolver as especificações-meta do produto, por meio das informações coletadas na fase de planejamento. As especificações orientam a geração de soluções e serve de base para que sejam montados os critérios de avaliação e de tomada de decisão utilizados nas etapas seguintes do PDP (ROZENFELD et al., 2006).

Segundo Rozenfeld et al. (2006), diferentemente da fase de projeto informacional que trata da aquisição e transformação de informações, o projeto conceitual consiste na busca, criação, representação e seleção de soluções para o problema, visando a obtenção da concepção do produto (ROZENFELD et al., 2006).

\subsection{Tênis paraolímpico Quad}

A Federação Internacional de Tênis é a entidade reconhecida oficialmente com autoridade para controle das regras oficiais do tênis em todo o mundo. Esta, regula não somente as regras oficiais do tênis para pessoas que não possuem deficiência, mas também de atletas que jogam tênis de cadeira de rodas, Quad ou divisão aberta dentro do esporte para reduzir o efeito da deficiência no desempenho esportivo (ITF, 2017).

Segundo o ITF (2017), os atletas que desejam competir na categoria Quad devem cumprir ao menos um dos requisitos a seguir:

- Função motora reduzida necessária para executar um serviço de sobrecarga;

- Função motora reduzida necessária para executar forehand ou backhand;

- Função motora reduzida necessária para manobrar uma cadeira de rodas manual,

- Incapacidade de segurar a raquete, exigindo a necessidade de tapar e/ou utilizar um dispositivo deapoio para jogar.

Segundo Bardales (2013) a importância do levantamento de informações de tecnologias existentes e de produtos similares consiste na geração de uma base consistente de informações sobre as funções de produtos similares e produtos que atendem as necessidades dos clientes. Essas informações influenciam diretamente na estimativa de custo das alternativas do produto em desenvolvimento, podendo incluir desde características do produto até propriedades do tipo de material e especificações (BARDALES, 2013). 
Agravando o problema apresentado, as soluções aplicadas atualmente envolvem pouca robustez tecnológica. Trata-se do uso de materiais, luvas, fitas, laços e colas para amarrar a mão à raquete de tênis. A carente inovação tecnológica aplicada neste contexto, apesar de uma falha, é também um incentivo para o desenvolvimento de novos produtos, que sejam capazes de habilitar e melhorar a experiência de jogadores de tênis, profissionais ou não, na modalidade paraolímpica denominada “Quad” (CARVALHO et al., 2005).

\section{METODOLOGIA}

A estratégia de pesquisa utilizada neste artigo foi o estudo de caso. Segundo Yin (2001), o estudo de caso pode ser utilizado em diversas situações tais quais estudos organizacionais e gerencias, como o deste artigo. A abordagem da pesquisa é majoritariamente qualitativa pois tem como objetivo a compreensão aprofundada de aspectos que não podem ser quantificados (GERHARDT, 2009). O estudo, por sua vez, tem como objeto o uso do modelo de referência de desenvolvimento de produtos de Rozenfeld et al. (2006) para a concepção de um produto que permite a reintegração de pessoas com tetraplegia incompleta ao esporte.

Nesse ínterim, foram utilizadas três técnicas principais para coleta de dados: levantamento de dados primários - que consiste na realização de entrevistas com os responsáveis pelo CETEFE e atletas paraolímpicos - levantamento de dados secundários - que abrange a realização de pesquisas científicas e busca em base de dados e metodologias que auxiliem no estudo - e observação direta - a qual consiste em visitas técnicas ao local para auxiliar na concepção do produto.

Para o desenvolvimento do produto foi preciso definir, primeiramente, o mercado atual, alvo e futuro e fazer os levantamentos de dados secundários e primários. Os dados primários foram obtidos, por meio da aplicação de um questionário com os funcionários da CETEFE em que foi aplicado o estudo, com fim de compreender a percepção dos jogadores de tênis com deficiência em relação a suas dificuldades. Já os dados secundários foram coletados para obter uma visão geral do problema de tetraplegia. Em seguida, foi possível estabelecer a viabilidade econômica e técnica do produto. 


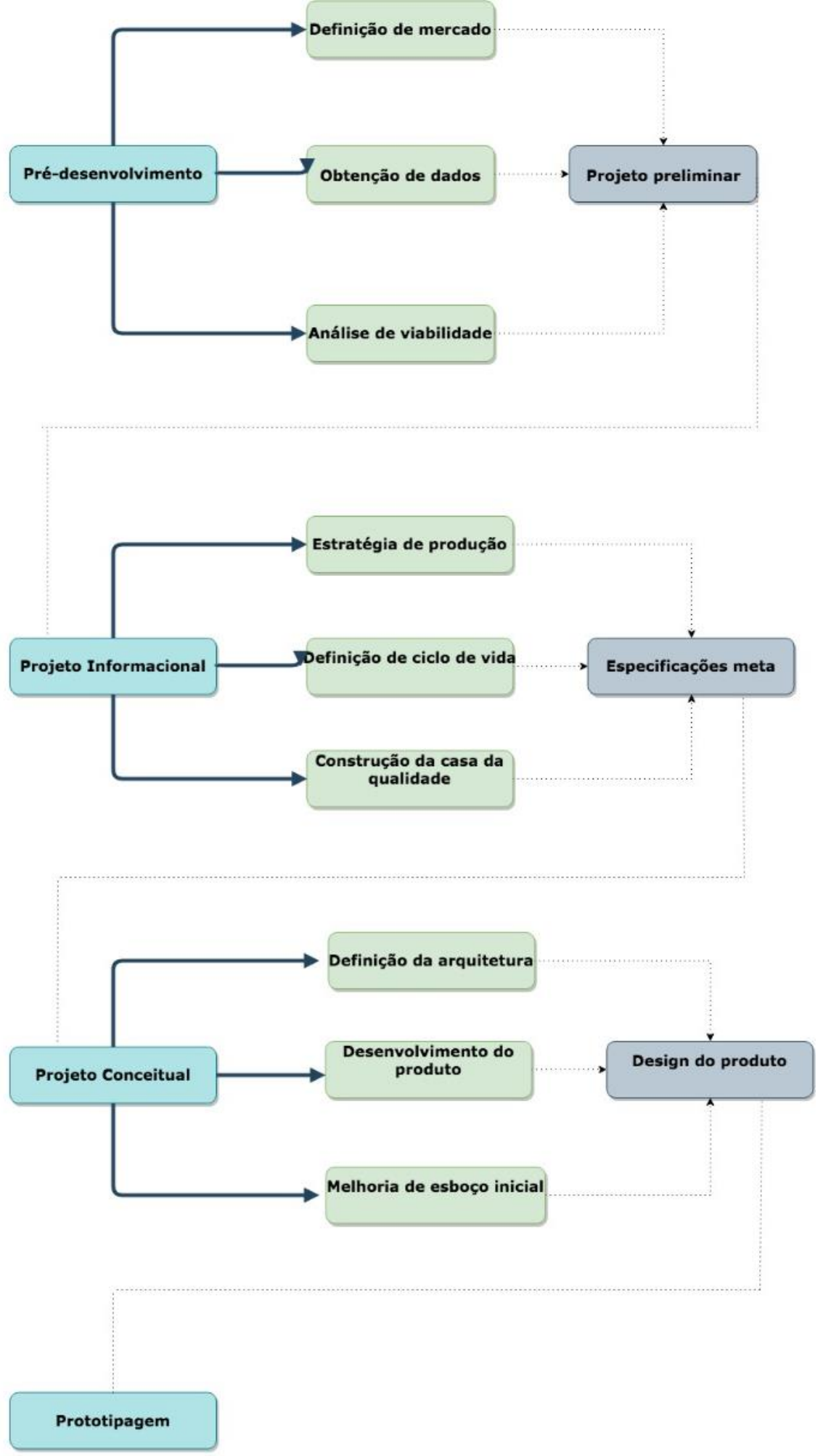

Figura 2 - Estrutura de desenvolvimento do projeto. Fonte: Autoria própria (2018) 
O projeto foi estruturado de acordo com a Figura 2, seguindo as etapas de prédesenvolvimento, projeto informacional, conceitual e prototipagem.
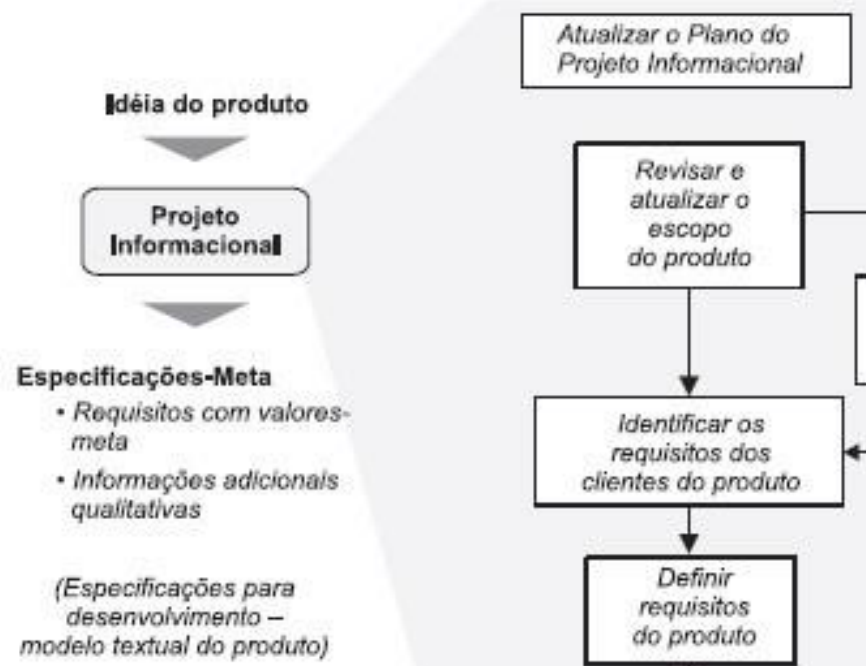
delo textual do produto

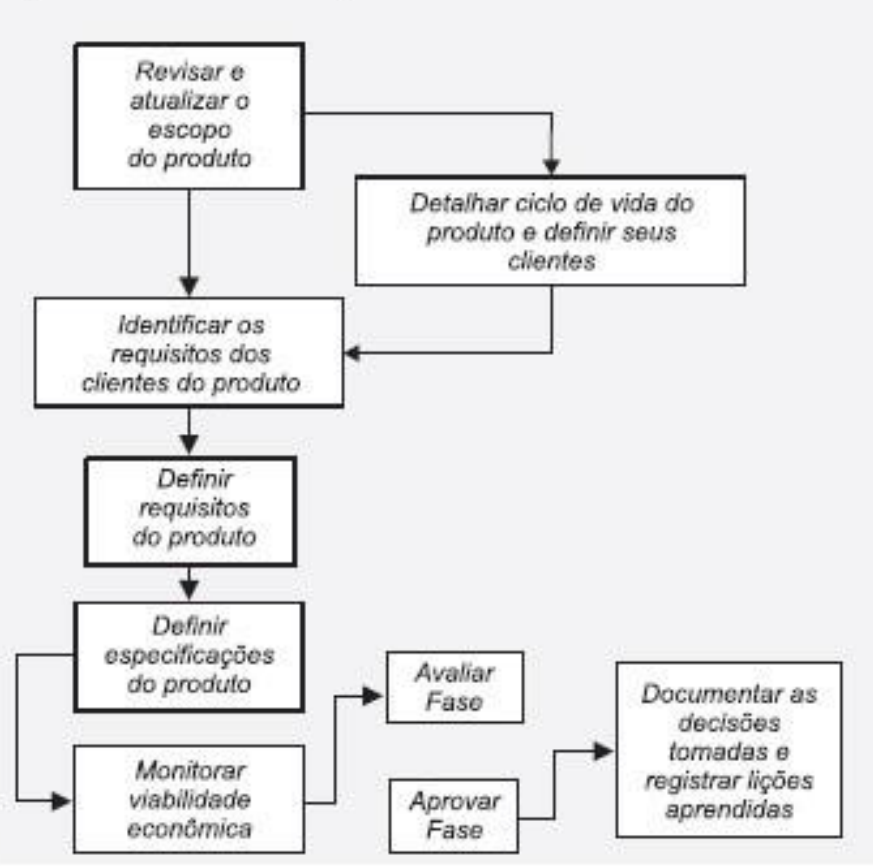

Figura 3 - Informações principais e dependência entre as atividades da fase de Projeto Informacional.

Fonte: Rozenfeld et al. (2006, p. 212)

A etapa de projeto informacional tem como principal objetivo definir as especificações meta. Para isso, foi necessário revisar o escopo do produto, estabelecer a estratégia de produção, ciclo de vida, identificar os requisitos do cliente para então determinar os requisitos de produto, as especificações meta e fazer uma revisão da viabilidade econômica do produto, conforme a Figura 3.

Para a coleta de necessidades dos clientes foram realizadas entrevistas com os funcionários do CETEFE e com atletas, além da aplicação de questionários e observação direta. Essas necessidades foram então convertidas para requisitos dos clientes. 


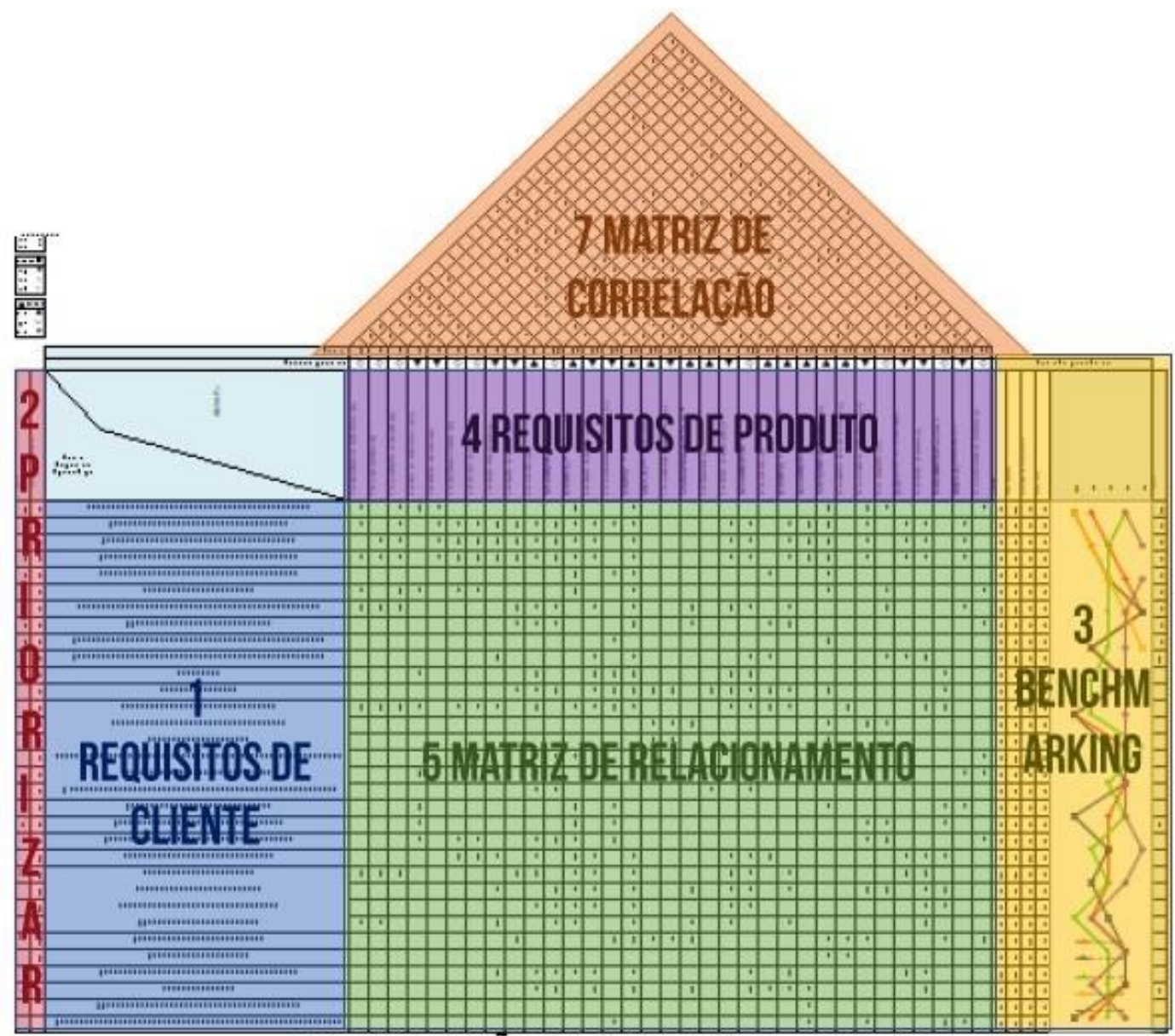

GQUHANTHICACAOADOSPRODUTOS

Figura 4 - Casa da Qualidade (QFD). Fonte: Autoria própria (2018)

A partir desta etapa, iniciou-se a construção da casa da qualidade QFD, como mostra a Figura 4. Após a conversão das necessidades do cliente para os requisitos do cliente, estes foram priorizados por meio do diagrama de Kano, Mudge e Pareto.

A terceira etapa da QFD, o benchmarking, foi realizada a comparação do produto em desenvolvimento com os produtos já existentes no mercado, como fita adesiva, fitas overgrip e um protótipo de empunhadura. Em seguida, foi possível definir os requisitos do produto e fazer uma matriz de relacionamento entre eles e os requisitos de cliente.

Em seguida, foi feita a quantificação dos requisitos de produto, ao multiplicar-se a importância dos requisitos de cliente pela matriz de relacionamento. Por fim, na QFD, foi obtida a matriz de correlação, que buscou identificar os requisitos de produto conflitantes e os requisitos positivos, a partir de uma análise par a par. 
Dessa forma, obteve-se as especificações metas do produto, definindo os requisitos de projeto, os objetivos, o sensor por meio que isso vai ser identificado e as saídas indesejáveis caso não se siga essas especificações. Essa etapa conclui o projeto informacional.

A etapa seguinte, o projeto conceitual, se divide em três fases: processo de criação e seleção da arquitetura, desenvolvimento do produto (componentes e subcomponentes) e análise de melhoria do esboço inicial.

A primeira etapa é apoiada pelos métodos de Modelagem Funcional, Matriz Morfológica (em conjunto com uma aplicação da TRIZ) e Matriz Indicadora de Módulos (MIM). Na segunda, embasada pela divisão e desenvolvimento dos Sistemas, Subsistemas e Componentes, foi realizada a seleção de materiais e uma prévia de possíveis fornecedores.

A aplicação da Análise de Valor e os Designs for X (DFx) resultou em propostas de melhorias para a concepção escolhida. Após isso, foi realizada a prototipagem e a revisão de viabilidade técnica e econômica.

\section{RESULTADOS E DISCUSSÃO}

A primeira etapa do projeto consistiu em realizar uma pesquisa para identificar o tamanho do mercado e quais pessoas seriam o público alvo do produto. Esta estimativa dos mercados atual, alvo e futuro é importante para definir se o produto é economicamente viável de ser produzido.

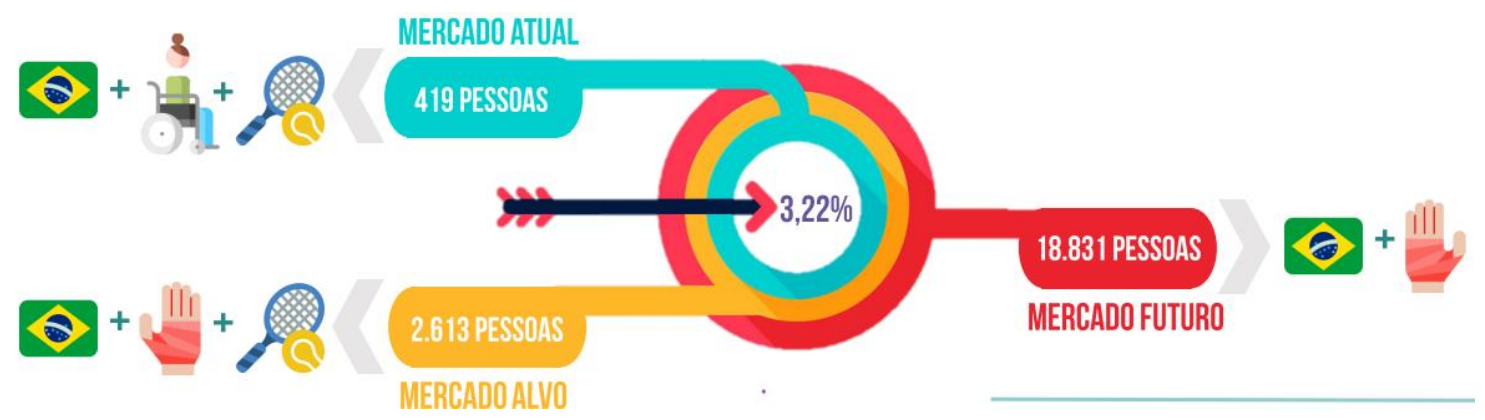

Figura 5 - Tipo de público e parcela do mercado. Fonte: Autoria própria (2018)

A Figura 5 apresenta uma projeção da quantidade de pessoas que seriam atendidas, considerando um market share de 3,22\% no mercado de tecnologias assistivas para o tênis.

O mercado atual (corresponde aos brasileiros com tetraplegia incompleta, caracterizada pela incapacidade de agarrar e transmitir força da mão à um objeto, sendo praticantes ou 
aspirantes ao esporte paraolímpico tênis) é extremamente restrito, atingindo 419 pessoas, o que acarreta em grandes probabilidades de a empresa não ser economicamente viável (BRAULT, 2012).

O mercado alvo corresponde a brasileiros que praticam ou possuem interesse no esporte tênis, mas que não necessariamente são tetraplégicos incompletos e sim pessoas que possuam qualquer tipo de dificuldade de agarra, aumentando o tamanho do mercado para 2.613 pessoas (BRAULT, 2012).

Por fim, como mercado futuro, entende-se que o produto não será destinado unicamente à prática do tênis tendo como público alvo qualquer brasileiro que possua dificuldade de agarra. Isto representa cerca de 580 mil pessoas, podendo ser atendidas 18.831 pessoas (BRAULT, 2012).

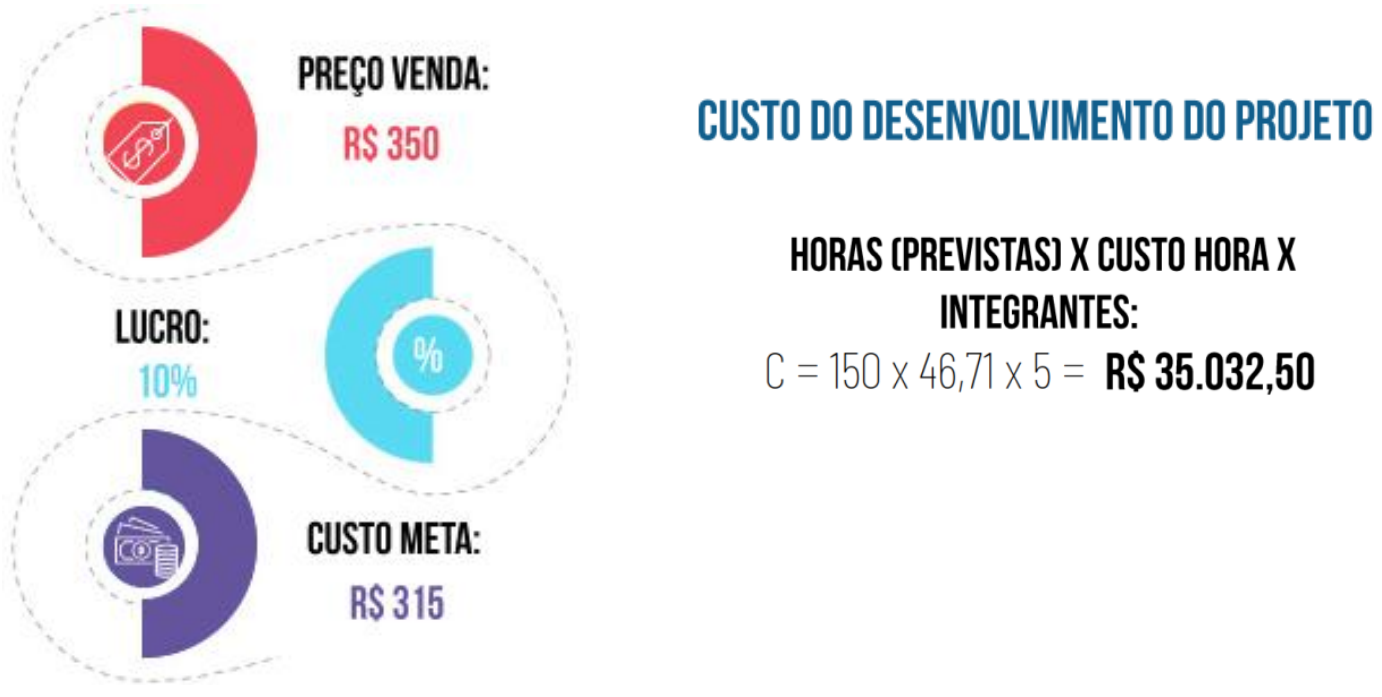

Figura 6 - Preço de venda do produto e custo de desenvolvimento do projeto. Fonte: Autoria própria (2018)

Os resultados financeiros estão representados na Figura 6, na qual pôde-se determinar o custo meta do produto, a margem de lucro esperada e o preço final de venda. Para determinar o custo de desenvolvimento do projeto, adotou-se o custo hora médio de um engenheiro de produção, a quantidade de membros na equipe e o total de horas necessárias ao longo de todo o projeto. 


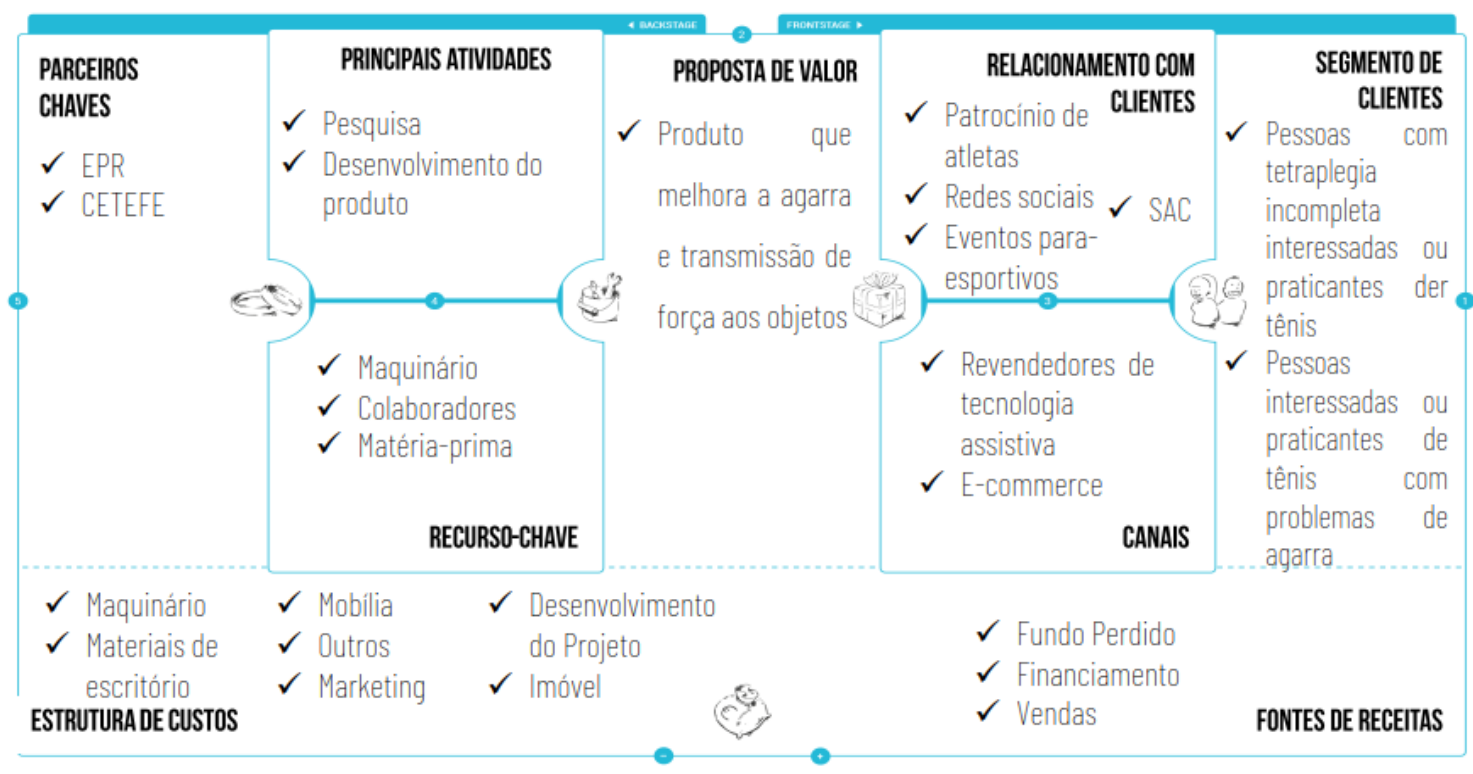

Figura 7 - Canvas de Negócios. Fonte: Autoria própria (2018)

O modelo de negócio viável, resultado do pré desenvolvimento do projeto, apresenta de forma gráfica e resumida: a proposta de valor, canais e relacionamento com o cliente, fontes de receita e estrutura de custos, além dos processos cruciais para o sucesso do negócio (parceiros, atividades e recursos-chave), apresentados na Figura 7.

\begin{tabular}{|c|c|c|c|}
\hline CÓDIGO & REQUISITO DOCLIENTE & $\begin{array}{l}\text { REQUISITO DO } \\
\text { PRODUTO }(A)\end{array}$ & $\begin{array}{l}\text { REQUISITO DO } \\
\text { PRODUTO (B) }\end{array}$ \\
\hline $1 . \mathrm{A}$ & $\begin{array}{l}\text { POSSUIR DISPOSITIVO QUE PRENDAA } \\
\text { MÄO DO JOGADOR }\end{array}$ & $\begin{array}{l}\text { 1.AA RESISTÊNCIA DO } \\
\text { DISPOSITIVO }(\mathrm{Pa})(+/ \text {-) }\end{array}$ & \\
\hline $1 . \mathrm{B}$ & $\begin{array}{l}\text { POSSUIR DISPOSITIVO QUE TRANSMITA } \\
\text { FORÇA À RAQUETE }\end{array}$ & $\begin{array}{l}\text { 1.BA FORÇA MECÂNICA(N) } \\
\qquad(+l \cdot)\end{array}$ & \\
\hline $1 . C$ & $\begin{array}{l}\text { POSSUIR DISPOSTIIVO QUE FACILITE A } \\
\text { AGARRA NA RAQUETE }\end{array}$ & $\begin{array}{l}\text { 1.CA COMPRESSÃO NA } \\
\text { RAQUETE }(\mathrm{Pa})(+/-)\end{array}$ & \\
\hline 2.A & SER LEVE & $\begin{array}{l}\text { 2.AAPESO DO DISPOSITIVO } \\
\qquad(\mathrm{KGF})(-)\end{array}$ & \\
\hline 2.B & $\begin{array}{l}\text { POSSUIR FORMATO QUE PERMITA A LIVRE } \\
\text { CIRCULAÇÄO DO SANGUE }\end{array}$ & $\begin{array}{l}\text { 2.BA PRESSÃO NAS } \\
\text { MÃOS(Pa) }(\cdot)\end{array}$ & \\
\hline $2 . \mathrm{C}$ & TER MATERIAIS CONFORTAVEIS & $\begin{array}{l}\text { 2.CA RESISTÊNCIAÀ TRAÇÃO } \\
\qquad(\mathrm{Pa})(+l-)\end{array}$ & $\begin{array}{l}\text { 2.CB MÓDULO DE YOUNG } \\
\qquad(\mathrm{Pa})(+/ \cdot)\end{array}$ \\
\hline 3.A & $\begin{array}{l}\text { SER FACIL DE ACOPLAR OS } \\
\text { COMPONENTES. }\end{array}$ & $\begin{array}{l}\text { 3.AATEMPO PARA ACOPLAR } \\
\qquad(\mathrm{t})(-)\end{array}$ & \\
\hline $4 . A$ & SER FÁCIL DE LIMPAR & $\begin{array}{l}\text { 4.AATEMPO DE LAVAGEM (T) } \\
\qquad(-)\end{array}$ & \\
\hline 4.8 & SER REUTILIZÁVEL & 4. BA VIDA ÚTIL (T) (+) & \\
\hline
\end{tabular}

Figura 8 - Conversão dos requisitos de cliente em requisito de produtos. Fonte: Autoria própria (2018) 
Com a aplicação da casa da qualidade, foram mapeados requisitos de cliente e de produto. A Figura 8 apresenta os 9 principais requisitos, já priorizados, do total de 32 requisitos mapeados. Estes foram sistematicamente considerados para então determinar as especificações meta do produto e gerar princípios de solução.

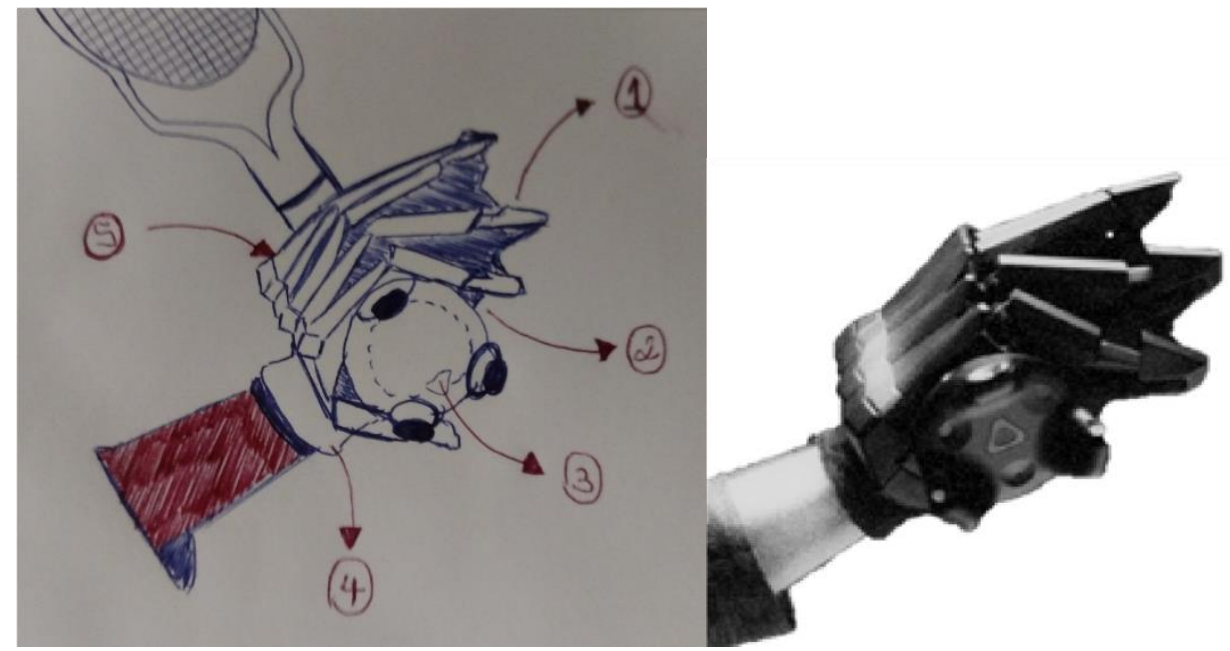

Figura 9 - Processo de criação e seleção de arquitetura. Fonte: Autoria própria (2018)
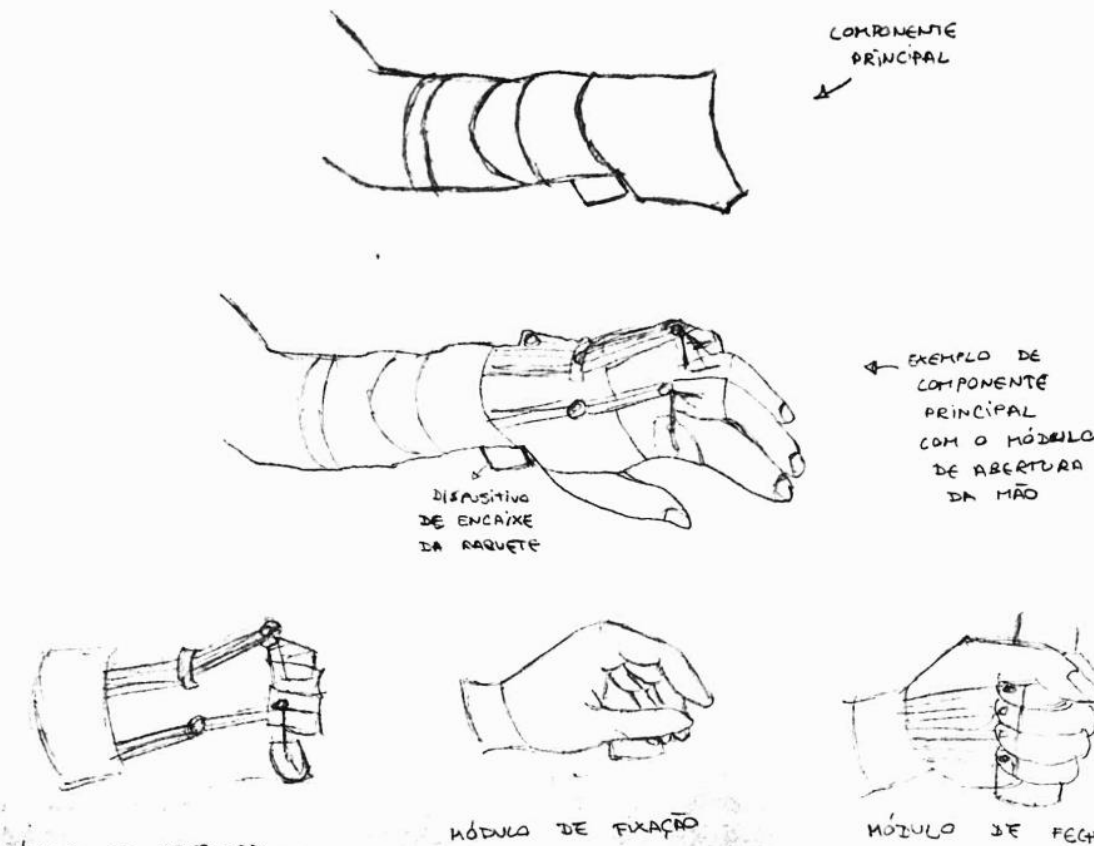

DA HADO

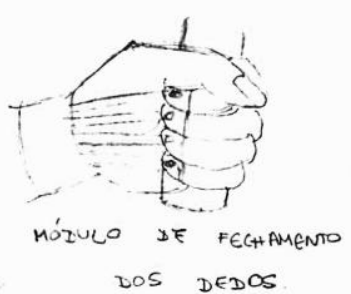

DA MÍ̄o

Figura 10 - Desenvolvimento do produto (componentes e subcomponentes). Fonte: Autoria própria 
Com a definição da estrutura funcional e da arquitetura do produto, foram geradas concepções de solução, representadas nas figuras 9 e 10. Para a seleção da melhor concepção, foi analisado o grau de atendimento aos requisitos de cliente apresentados. Com a concepção escolhida, esta pôde ser então melhor detalhada.
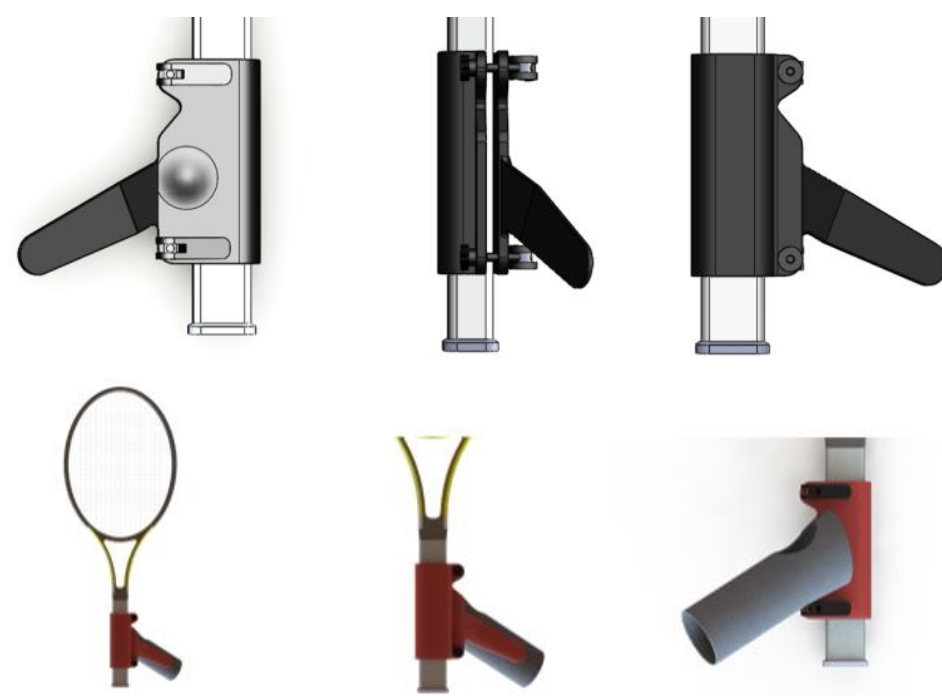

Figura 11 - Protótipo em CAD renderizado. Fonte: Autoria própria
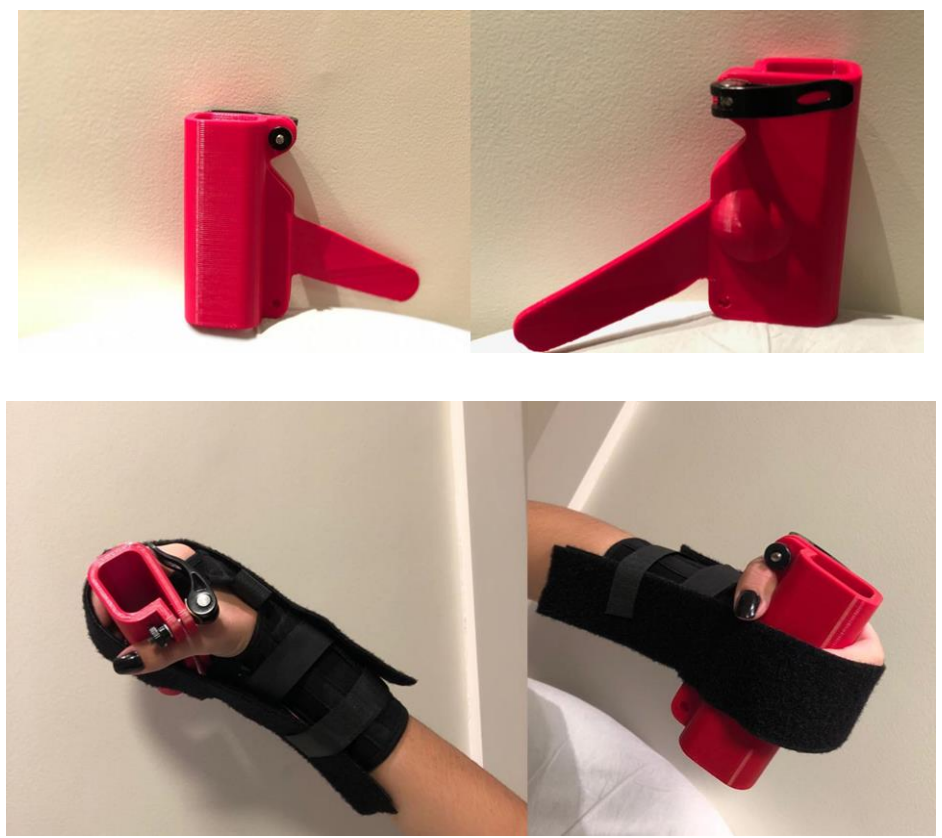

Figura 12 - Protótipo do componente principal impresso em 3D. Fonte: Autoria própria.

Com o objetivo de obter uma melhor compreensão da modelagem escolhida e de suas respectivas funções, foi realizada uma prototipagem do produto em CAD (Figura 11) bem como 
impresso o componente principal do produto (módulo de encaixe da raquete) em uma impressora 3D para testes com o usuário (Figura 12).

\section{CONCLUSÃO}

Tendo em vista o conteúdo exposto e o objetivo da pesquisa de desenvolvimento de um projeto de produto de tecnologia assistiva para jogadores de tênis tetraplégico, conclui-se que o projeto de produto é satisfatório, uma vez que demonstrou a lógica utilizada em sua construção, avaliação e concepção baseada no processo de desenvolvimento de produtos.

A validação do protótipo obtida através de entrevistas com os membros do CETEFE demonstra a adequação das características básicas de qualidade técnica e ergonômica do protótipo e a concordância do referencial teórico utilizado em sua construção. O protótipo foi impresso em 3D, possui desenho modular e capacidade de portabilidade, promovendo segurança e acessibilidade funcional para praticantes de tênis tetraplégico.

O estudo identificou que apesar do restrito mercado alvo, as soluções aplicadas atualmente envolvem pouca robustez tecnológica e comprometem a participação, a motivação e a evolução esportiva dos atletas. Observou-se que o protótipo além de ajudar na sustentação da raquete, promove a proteção dos membros superiores, alvo de lesões até mesmo em atletas não deficientes. Além disso, foi identificado que para a concepção do produto devem ser consideradas características antropométricas dos usuários, nível de tetraplegia e os regimentos esportivos específicos da modalidade. A realização de entrevistas indicou as necessidades explícitas e implícitas dos praticantes e os questionários aplicados mostraram-se eficazes na identificação de óbices muscoesqueléticos relacionados à dor.

O produto se encontra em um nível de maturidade tecnológica (TRL) 2, no estágio de atividade inventiva e possui um alto nível de objetividade e reprodutibilidade, possibilitando a utilização da concepção em trabalhos futuros. Nesse ínterim, pode-se analisar que a pesquisa apresentou informações técnicas e configurações práticas na concepção do produto possibilitando a reintegração de pessoas com tetraplegia incompleta na modalidade de tênis Quad.

\section{REFERÊNCIAS}

ALDEN, Chris, and Amnon Aran. Foreign policy analysis: new approaches. Routledge, 2016. 
BARdales, A.. Modelo De Estimativa De Custos Para As Fases Iniciais Do Projeto De Produto Mecatrônico. Dissertação de Mestrado, UnB, 2013.

BRAULT, Matthew W. et al. Americans with disabilities: 2010. Washington, DC: US Department of Commerce, Economics and Statistics Administration, US Census Bureau, 2012.

CALDWELL, Mary, and Arthur Jason De Luigi. Wheelchair Dance Sport. In Adaptive Sports Medicine, pp. 171-179. Springer, Cham, 2018.

CARVALHO, Alzira Alves de Siqueira. Miopatias. V Simpósio de Hipertermia Maligna. Revista Neurociências. São Paulo, 2005.

CONFORTO, Edivandro Carlos; AMARAL, Daniel Capaldo; SILVA, SL da. Roteiro para revisão bibliográfica sistemática: aplicação no desenvolvimento de produtos e gerenciamento de projetos. Trabalho apresentado, n. $8,2011$.

GERHARDT, Tatiana Engel; SILVEIRA, Denise Tolfo. Métodos de pesquisa. Plageder, 2009.

HART, Oliver, and John Moore. Default and renegotiation: A dynamic model of debt. The Quarterly Journal of Economics113.1, 1998: p. 1-41.

ITF, INTERNATIONAL TENNIS FEDERATION. Quad Draw Player List, 2018. Disponível em: <https://www.itftennis.com/media/290175/290175.pdf>

ROZENFELD, Henrique et al. Gestão do desenvolvimento de produtos: uma abordagem por processos. São Paulo: Saraiva, 2006.

SHAW, Jonathan. A schema approach to the formal literature review in engineering theses. System, v. 23, n. 3, p. 325-335, 1995.

ULRICH, K. T.; EPPINGER, S. D. Product design and development. New York: McGrawHill, 2004.

USPTA. Do You Want To Be a Player, B. T., ANYONE, T., Pro, U. S. P. T. A., Mills, J., Waite, T. T. R., ... \& Murray, J. TENNIS EQUIPMENT TIPS. 2018. 